\title{
Data Agents: Promoting Reflection through Meaningful Representations of Personal Data in Everyday Life
}

\author{
Maria Karyda \\ first.last@aalto.fi \\ Aalto University, Espoo, Finland
}

\author{
Elisa D. Mekler \\ first.last@aalto.fi \\ Aalto University, Espoo, Finland
}

\author{
Andrés Lucero \\ lucero@acm.org \\ Aalto University, Espoo, Finland
}

\begin{abstract}
Visual and physical representations of historical personal data have been discussed as artifacts that can lead to self-reflection through meaning-making. However, it is yet unclear how those two concepts relate to each other. We focus on meaningfulness, a part of meaningmaking that relates to feelings. In this paper, we present three projects where mundane objects, our data agents, are combined in meaningful ways with personal data with the aim to trigger reflection by placing a person's individual experience of data in relation to others'. To identify relationships between self-reflection and meaningfulness we use Fleck and Fitzpatrick's framework to describe the levels of reflection that we found in our projects and Mekler and Hornbæk's meaning framework to define the depth of reflection. We conclude with a discussion on four themes in which we outline how data agents informed the intersections between our central concepts. This paper, constitutes a first step towards unpacking those relationships and invites for further explorations by the HCI community.
\end{abstract}

\section{CCS CONCEPTS}

- Human-centered computing $\rightarrow$ Empirical studies in HCI.

\section{KEYWORDS}

Meaningfulness, self-reflection, data representations, personal data

\section{ACM Reference Format:}

Maria Karyda, Elisa D. Mekler, and Andrés Lucero. 2021. Data Agents: Promoting Reflection through Meaningful Representations of Personal Data in Everyday Life. In CHI Conference on Human Factors in Computing Systems (CHI '21), May 8-13, 2021, Yokohama, Japan. ACM, New York, NY, USA, 11 pages. https://doi.org/10.1145/3411764.3445112

\section{INTRODUCTION}

Previous work has explored people's relationships with their personal data through a diverse set of artifacts of different forms and formats $[35,40,41]$. Necessarily, relating to the data through those artifacts requires a process of meaning-making. Through that process the person aims to understand the information they are presented with (make-sense of the external world), and also, to often critically engage in a process of self-reflection. In particular, it has

\section{(c) (i) (-)}

This work is licensed under a Creative Commons

Attribution-NonCommercial-ShareAlike International 4.0 License.

CHI '21, May 8-13, 2021, Yokohama, Japan

(C) 2021 Copyright held by the owner/author(s)

ACM ISBN 978-1-4503-8096-6/21/05.

https://doi.org/10.1145/3411764.3445112 been argued [17] that the process of engaging with personal data through alternative forms can help individuals arrive at new found understandings about their data but also about thyselves [42]. For example, on one hand, personal informatics tools [11, 23, 24, 31] support engagements with (biometric) data by generating visualizations of a person's performance (historical data) that later, through the process of self-reflection, can motivate behavioral change. On the other, data physicalizations $[17,28,30,36]$ invite people to engage with their personal data in visual, haptic and sonic waysthus to explore their data by involving more than one of their senses. This exploratory engagement with data in material forms is meant to trigger meaning-making processes commonly through unforeseen associations between data and form, which may lead to self-reflection. Generally, in current literature on personal informatics and data physicalizations, there is a clear interrelation between meaning-making and reflection however, it is yet unknown how those two elements relate to each other [26].

Notably, most works on data physicalizations have focused on meaning-making processes with only few examples focusing on how people make sense of data through meaningful representation (relating the data to the inner world). Bruner [6] argues that "meaning-making describes the process wherein one imbues a particular event or phenomenon with a sense of personal significance, whereas subjective meaningfulness reflects the experience of feeling as though something matters." In that statement, meaningfulness is argued to be part of meaning-making that relates to the subjective experience of meaning through feelings. This indicates that in order to study meaningfulness in the context of physical representations of personal data, one should aim at first understanding the human experience of data - in essence, to inquire into how people understand personal data representations through feelings.

To this end, we present three different projects that we use as leverage to articulate how meaningfulness supports or even enhances people's reflective processes. To do so, we have used a critical design approach [10] in which we enabled combinations between people's personal data and meaningful everyday objects such as gifts. All three projects presented in this paper aimed at challenging the status quo of the use of personal information in different contexts and to provoke reflexive thinking. Our data artifacts were meant to enable agency and actively invite meaningful and reflexive engagements on behalf of the data. To describe the central data artifacts of those three projects we use the term data agents. Data agents are mundane objects combined with people's personal data in meaningful ways. Their role is to trigger self-reflection while people revisit their understandings about their personal data and data practices. In that context and to achieve that, data agents place a person's individual experience of data in relation to others'. 
In our work we perceive and deal with meaning and reflection as subjective experiences. To find relationships between self-reflection and meaningfulness, we employ Fleck and Fitzpatrick's [12] framework to describe different levels of reflection that took place during our projects, and Mekler and Hornbæk's [26] meaning framework to help us articulate how data agents prompted deeper levels of reflection. Hence, we pose the following research questions: How can meaningful representations of data facilitate self-reflection? How do self-reflection and meaning-making relate to each other?

This paper contributes a first step towards understanding how meaningfulness and self-reflection relate to each other in the context of personal data representations. We acknowledge that presenting any relationship between these very complex concepts is a very demanding task. Therefore, through the selection of those two frameworks $[12,26]$ we aim at simplifying and unpacking how we can understand the relationship of those central concepts. In addition, the work we present in this paper is an invitation for the HCI community to challenge, scrutinize and extend our findings.

\section{DATA REPRESENTATIONS, SENSE-MAKING AND REFLECTION}

In this section, we discuss how previous work on personal informatics and data physicalization have dealt with aspects of self-reflection and meaning-making. Next we dig deeper into the role of meaningfulness and its relationship to self-reflection.

\subsection{The Role of Reflection in Personal Informatics}

In personal informatics reflection is often described as "providing increased self-knowledge" [4]. Within HCI there are two fundamental perspectives on personal informatics, a technology-centric [24] and an experience-centric view [11,31], with some authors [e.g., 8] acknowledging the value of both without taking a clear stand. While complementary, both perspectives have different starting points, which is also mirrored in how they understand reflection.

Choe et al. [8], for instance, understand reflection as personal insights. In their work they combined visualization and a thinkaloud approach to capture participants' insights upon looking at the visualizations of their personal data. However, Choe et al. do not consider the idiosyncrasies of people. Not everyone is stimulated by the same visual aids. Their work aimed to capture people's self-reflections, however, the parameters of the study potentially minimized the type of insights the authors would get if this study had been conducted in the wild. That, is acknowledged by the authors however, what exactly reflection is, is not described in the paper.

Li et al. [24] appear to have an instrumentalist view of selfreflection. The authors identify two phases of reflection, which they name discovery and maintenance. "The distinction between the phases is important because it highlights the need for personal informatics tools to support different information needs." Self-reflection is identified as an element that can raise people's awareness about their behavior, motivate behavioral change and help people make better decisions. The authors argue that the value of reflection is affected by how and what data a person collects. They suggest that Ubicomp technologies can improve how data is collected and increase the types of data that are collected to improve self-reflection. Differently, Rooksby et al. [31] coined the term lived informatics, which refers to the use of activity trackers in everyday life in particular with a focus on a fraction of the personal informatics field that relates to domains such as life-logging. The same authors suggest that to design personal informatics tools we should not ignore how personal tracking is enmeshed in everyday life. As the authors argue "Little tracking was being done for the sake of building up a stock of data about life" where people interweave several trackers together probably for the purpose of self-reflection. This is in line with Baumer et al.'s [4] definition of reflection, which suggests that reflection includes "reviewing a series of previous experiences, events, stories, etc., and putting them together in such a way as to come to a better understanding or to gain some sort of insight." The latter indicates some kind of synthesis of information that a person or a group of people would perform, which will then facilitate some kind of transformation [3] in their fundamental understanding about the information they were initially presented with.

Recent work by Elsden et al. [11] describes a design space, the quantified past, which supports the long-term and retrospective use of personal informatics. In the same article, the authors propose "an opportunity to offer new modes of experiencing amorphous and malleable data" for remembering and reflection. Their understanding of designing for historical data and life-logging is in line with Rooksby's [31] concept of lived informatics inviting designers to consider the experience of everyday life when designing for personal informatics and life-logging of data. Thus, while Elsden et al. share Wright's and McCarthy's [43] experience-centric view of technology, they invite designers to produce personal informatics tools for "evocative and emotional engagements with data", which may offer to people a different rhythm for reflection. Elsden et al. go on to suggest that "new modalities and materialities" of personal data can support different kinds of sense-making compared to what personal informatics tools can currently offer.

In our work, we use Fleck and Fitzpatrick's [12] framework to identify how reflective thinking occurred in our projects, which we also present in Table 1. In their framework, critical reflection links to relating one's experience to wider social and ethical implications forming the highest and least common level of reflection. The lowest levels (i.e., 'revisiting / non-reflective description' and 'reflective description') refer to mentally revisiting one's experiences, but with little to no consideration of alternative explanations or viewpoints. 'Dialogic (or dialogical) reflection' denotes both the looking for relationships between one's knowledge and experiences, as well as the exploration of alternate hypotheses and different perspectives. That may then result in 'transformative reflection', that is, the altering of one's assumptions and/or behavior.

\subsection{Sense-Making in Data Physicalization}

Data physicalizations [17] are material manifestations of data that can support this different kind of remembering (as suggested by Elsden et al. [11]) and reflection by involving multiple senses together such as hearing, touch and sight. In addition, data physicalization reflections may occur through design iterations $[2,13]$, construction 
Table 1: Levels of reflection outlined in Fleck and Fitzpatrick's framework [12].

\begin{tabular}{ll}
\hline R0 Reminiscing & $\begin{array}{l}\text { Remembering an experience without further reflection } \\
\text { R1 Reflective Description }\end{array}$ \\
R2 Dialogic Reflection & $\begin{array}{l}\text { no alternate explanations explored } \\
\text { Looking for relationships between instances of } \\
\text { experience, cycles of questioning and interpreting }\end{array}$ \\
R3 Transformative Reflection & $\begin{array}{l}\text { Reflection with the intent to change one's behavior, gain new insights, } \\
\text { or reconsider personal assumptions }\end{array}$ \\
R4 Critical Reflection & Relating one's experience to wider social and ethical implications \\
\hline
\end{tabular}

in small and large scale $[29,40]$ and by comparing data with others [29]. Barrass [2] has created sonifications of his personal data. In an exploration that borrows characteristics from autobiographical design, Barrass fabricates the Hypertension Bowl. In that example the interaction with the objects triggers novel associations between data and sound. The author went through several iterations of the singing bowl resulting into one that would produce the most pleasing sound. From this we can speculate that reflection may occur during an iterative design process from one model to the next, and then to the interaction with final sonification. Frick [13] is an artist who creates installations of her sleeping patterns, organises the data in small wooden or glass tiles and arranges them in space. The practice [32] of arranging different information in space acts as a trigger for reflection. In that case, the body becomes the main agent aiming to discover the nuanced self through a haptic experience. In a smaller scale, Huron et al.'s [16] constructive visualizations builds upon constructive theories of learning, arguing that physical data construction is deeply connected with learning-and to selfreflection-since reflective thought is common in education and learning [32].

Involving the body as an active agent in data physicalization has been a way through which people can synthesise past data to then reflect on it both individually [16] and also in some cases collectively [29]. In Data-things [29] people were invited to explore through knitting their personal data. A visualization was formed by each participant through sensors placed on their knitting needles. Later, the same people were involved in the digital fabrication (i.e., laser cutting) of their visualizations during which, they reflected on their data through construction, as well as by comparing and discussing with the rest of the group. That project illustrated the role of social aspects in provoking self-reflections. In that context, data was analysed among different knitting practitioners who were juxtaposing their 'data-things.' In the same article, another case study is presented following again a participatory approach where different people were given a physicalization of their tweets during a conference. The participants of that study compared the physicalizations with each other. In both examples, 'data-things' became tickets-to-talk about data with people that shared similar motivations e.g. similar interests in a practice (e.g., knitting) or interest in an event. Differently, in our work our designs are viewed as 'agents' in everyday life, which may give the opportunity for people to share and interpret their personal data in different environments with different people and therefore, actively shape their understanding of the represented data.

\section{MEANINGFULNESS AS A PATH TO REFLECTION}

Close to sense-making of data physicalizations through artifacts that can support "evocative and emotional engagements with data" [11], there are several other explorations in HCI that have uncovered evocative, enchanting and meaningful experiences between people, data and artifacts. Carpenter [7], for example, explored meaningfulness in the context of IoT devices. In particular, she describes "manifestations" of meaningfulness as physical characteristics that may enable meaningful experiences. Specifically, devices are thought to become most meaningful if they are non-screen, tangible, craftsbased and everyday objects. Next, we present examples from HCI that manifest those characteristics. The following examples are artifacts that became meaningful for people in different ways while the same artifacts were representing-literally or in abstract waysdata people could relate with. For people, seeing or even interacting with that data led to reflections about themselves.

A notable example is Thomas et al.'s [38] work on reflection through digital remediation of personal information on social media. This work focuses on aspects on reminiscence and reflection through curating and embedding meaningful digital content of people's social media on a printed book, on a triptych consisting of three printed framed pictures, and a film compiled out of information that can be found on a person's different social media platforms/profiles-our focus is on the first two tangible media. Certainly, in that project the process of selecting what data to display/curating on different media is in itself an act of selecting what is meaningful and important to an individual. In a wider context, Koeman et al. [21] deployed voting devices and visualizations in an attempt to enable community-wide participation. In particular, that project was conducted in Mill Road in the UK, a street divided by railway bridge, which reflected also a divide between the communities on each side. Thus through a simple voting system in different shops and cafes people engaged in answering topics of relevance for both sides of the street. Through visualizations on the pavement that illustrated the results of voting, people then reflected on their community as a whole beyond the divide. That project was meaningful for people as the visualizations were enmeshed into their daily lives in a simple way and allowed for ongoing reflection through dialogue with the shop owners and people around them.

Selby et al., [33, p. 2] explored the idea of Experiential Manufacturing that "aims to create more intrinsic, and aesthetic experiences of biographical data, and to provide meaning beyond the recall of information." To explore this, they created the Earthquake Shelf, a shelf that vibrated to demonstrate real time earthquake data from a 
Table 2: Mekler and Hornbæk's five components of meaning and their definitions [26].

\begin{tabular}{ll}
\hline Connectedness & Connected to the self and the world \\
Coherence & Making sense of one's experiences and how they fit into one's life \\
Resonance & Feeling that something is right \\
Purpose & Sense of core goals, aims and directions \\
Significance & Enduring value and importance \\
\hline
\end{tabular}

remote location. The prototype was a provocation and its purpose was to evoke the reconstruction of memories and "their association with 'new' objects through the action of the device." However, that was not successfully facilitated through the prototype, as it had negative connotations, what Lupton [25] has described as "data visceralisations, where data do not make sense because they do not feel right." Mekler and Hornbæk's framework [26] suggests that the data and the shelf may not have resonated with participants, nor could they make sense of it upon further reflection, because it was not sufficiently connected to their self and lacked other links they could personally relate to (e.g., the lived experience of a person who sensed the earthquake). The prototype facilitated a meaningful connection between the person who used it and his own community since the shelf was representing live time data from that person's home country. Thudt et al., [40] investigated the construction of personal data physicalization at people's homes and how self-reflections may occur in that context. The authors argue that reflection already started before entering the construction process, the same process allowed focusing on personally meaningful aspects and enabled personal mappings of qualitative experiences. The types of self-reflection that were identified in this work were related to predictions, reminiscing, testing new strategies, capturing accomplishments, sticking to goals, negative responses e.g. stress and associating data readings to personal values. The process of construction and crafting is an inherently reflective process [32] while the body becomes the protagonist of the interaction and this is when the individual can perhaps focus on values and personally meaningful aspects.

In Data-Craft [39], Thudt created ceramic tableware that incorporated on their surfaces representations of meaningful data related to the first author's partner and a friend. The purpose of this work was to create meaningful mementos for habitual use that would "support personal and joint reminiscing." While the ceramic objects represent data that is meaningful for the first author, there is a big disconnect between the experience behind the data and the artifact of representation. Also, in their paper it is not clear how and if the objects became triggers for joint reminiscing and/or reflection. However, what appears to be significant in this project is how meaningful personal data becomes part of everyday life through mundane artifacts, in that way, data is placed in the core of the lived experiences.

Mekler and Hornbæk [26] suggested that data representation artifacts may not only support sense-making, where people attempt to comprehend the data, but also aim at making data "meaningful." According to their framework on the experience of meaning, this "meaning-making" encompasses self-reflection on how the data relates to people's personal goals, figuring out what is important to them, as well as making sense of how the data relates to oneself and one's relation to the world. In turn, such meaningful experiences may inspire further reflection. This is nicely illustrated through the above examples. With regards to data physicalization, Mekler and Hornbæk [26] highlight the work of Houben et al. "to make the data more meaningful by [...] help(ing) users become interested in, and understand the data streams more in the context of their own lives" [15, p. 1610], where the physicalization "helped the participants to think and reflect on the data changes and made it more meaningful when looking at the data provided" [15, p. 1616]. In Table 2, we present the five components of meaning as outlined by [26].

Overall, the above examples show that personal informatics, and data physicalization efforts in particular, strive to foster reflection and somehow yield personally meaningful insights. However, in the majority of the works this remains more an assumption or an afterthought, as reflection and meaningfulness are rarely explicitly considered. This raises questions on how that meaning-making process actually leads to reflection. Moreover, we speculate that this is so since those examples have not been really used nor experienced (in the wild) outside the setting of those studies to evaluate their impact. That raises the questions, what will happen when those materializations of a quantified past [11] will be incorporated into everyday life, but also how will this be achieved [25]. For that, we employ the lens of meaningfulness [7,26] and Fleck and Fitzpatrick's reflection framework [12] to explore how manifestations of data become part of the everyday life and enable reflections in- and on-action [32].

Next, we present our three case studies. In each, we illustrate our findings and briefly outline how each project relates to reflection and meaningfulness. In our discussion, we use the two frameworks to outline potential relationships between meaningfulness and reflection. Thus, all three studies that will be presented below are critical design projects as their purpose is to challenge the status quo of digital data practices and provoke critical thinking. More specifically, the first study, challenges the presentations of self on social media - how we appear online through information; the second, makes a critique towards the inconsistencies between biometric data and lived experiences; and the last, shows how can we use personal data to sensitize others over phenomena around the world.

\section{RESEARCH APPROACH}

In our studies, we undertook a Research through Design approach (RtD) [22]. Koskinen et al. (2011) divided RtD into three approaches lab, field and showroom. The projects presented in this paper are related to the showroom approach. The showroom approach builds on contemporary art and design traditions that find their outset in the Royal College of Art in London [10]. The aim of this approach 


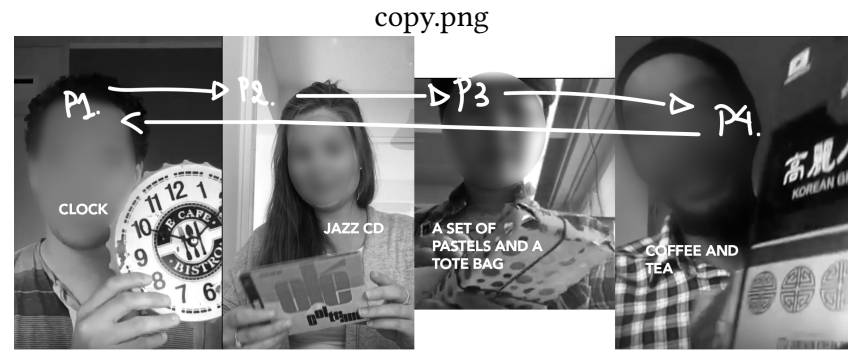

Figure 1: From left to right we present the flow of who gave a gift to whom as well as the type of gifts each of our participants received.

is to generate stories rather than facts. Through provocation it creates debate and raises awareness on critical societal issues. It does so through design interventions, typically by using material artifacts, prototypes. Those artifacts have been typically displayed in artistic exhibitions such the example of the Audio tooth implant [18] by Loizeau and Auger, but there are also examples of critical design explorations that have been used even within organizational settings e.g. provotypes to enable organizational change [5]. While in our first two projects our data agents are designed for use under certain contexts in which they are tested, our last case, is presented more as an 'exhibition' piece.

\section{THE CASE STUDIES}

The data agents present here are all imbued with a representation of personal data used to construct the personal narrative, which then results in triggering unforeseen reflection in relation to data and self. Dunne [9] argue that the purpose of critical design is to invite the viewer into a world of ideas rather than objects and to make their audience think. This is something that all three projects aim to achieve. Additionally, in critical design there are no methodological directions [1], those are only implied through the following words: transgression, provocation, satire and the staging of dilemmas. As such, the third project is not discussed in relation to participants' insights, its critical rhetoric is articulated through the juxtaposition of two different social realities. It is important to highlight that the data represented through our objects were inspired by people's idiosyncrasies and lived experiences. In the following projects we use pseudonyms to refer to our participants.

\subsection{Gifting a Stranger: Online Presentation of Self}

The first project presented here investigates how available online data can be used to pick a meaningful present for a stranger and by extension how do people make-sense of those gifts [19]. As argued by Sherry [34], a gift reflects the perception of the donor and the recipient regarding the identity of self and other. In our project, the concept of gift-giving as a meaningful gesture that represents both giver and receiver, was used to help people reflect on how others perceive their available online data. Our aim was that the gifts would trigger reflections about the self through the lens of others yet represented in a material form.
For our project, we recruited and asked four individuals, two women and two men, of different cultural and professional backgrounds, to buy a gift for a stranger-one of the other participantsinspired by the information that they could find about one another on social media. To do so, they received instructions in the form of individual invitations that in appearance resembled gifts (see Figure 2). The invitations included, (a) information of the person each participant had to look up online, (b) the information that their gifts should cost up to 10 Euros, and (c) instructions on how to document the process of opening the received gifts. The purpose of the latter was to capture our participants' first reactions and reflections when opening the gifts. Capturing those first thoughts was essential to understand how those gifts made them reflect. All participants gave and received a gift, but they did not personally exchange them. In Figure 1, you can see the order in which the gifts were given. To conclude, we conducted individual semi-structured interviews with the four participants. There, the participants were asked to reflect on their experience both as givers but also as receivers of the gifts. Here, we focus on the reflections of our participants as receivers of the gifts, as we were particularly interested in how they reflected on the material representations of their personal data.

Upon receiving their gifts, all participants were both positively and negatively surprised, as they could not comprehend where their gift-givers had found the data that informed the gift choice. Some of the gifts were very personal indicating that the gift-giver had succeeded in finding the right information online. For instance, Alice received a CD of Jazz music from Jack who found that Alice used to sing in a choir when she was younger. This information was found through a link on Alice's Linked-In profile. More specifically, Jack said, "I took two small pieces of info and then started walking around places." Nevertheless, Alice was surprised, as she could not figure out where Jack had found that information, "I wonder how my social media and my profile tells about music because I was just wondering if the only thing that comes through is my work because I guess that's the first thing that comes out when you Google me." After reading the instructions she received with the gift where we asked for her first impressions with the gift she said, "Well I wouldn't have expected a CD, not at all [...] although there is an old story of me online playing the saxophone, perhaps that's the connection?" In that moment she was reflecting on her social media presence. That indicated that our participants did not always know what was online publicly available about them.

This was also the case with Blair, who was surprised by how personal the gift she received was. Blair's gift was a set of pastel colors, as she is a mother of a young child and in her work as a designer she also works with children. This particular gift also included a card, which made it even more personal. Note that our participants chose their gifts based solely on the information that was public on the other person's profile. The process of trying to make sense and remember what information they individually had online, made participants reflect on their online presentation, which brought up aspects of privacy but also awareness and control on what is available online. The gifts have stayed with the participants for three years now.

In this project, the concept of gift-giving, which resembles a social bond between two or more people, was taken out of context to facilitate a playful experiment about personal data. While the 


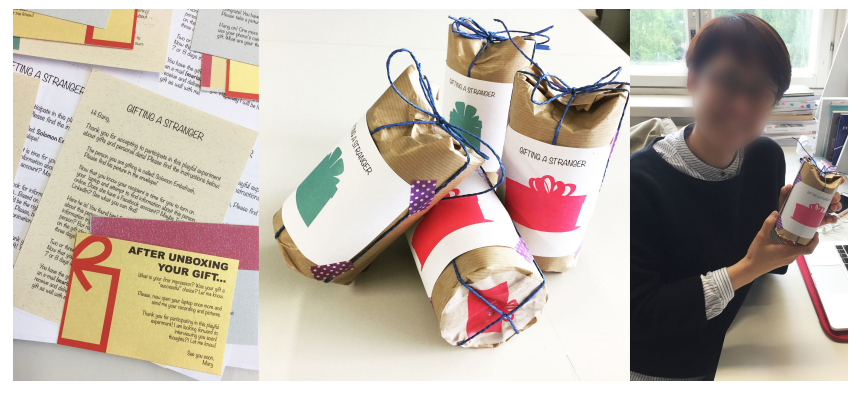

Figure 2: From left to right we present the content of the instructions, the package with the instructions and one of the participants when she received the instructions.

gifts were bought by strangers, the social structure that the notion of gifting encapsulates nevertheless manifested in the way people wrapped their gifts, as well as their thoughtfulness in choosing the gift. In this project, the aspect of getting something meaningful like a gift for someone, was the trigger for reflective thinking on the information people had online about themselves. The gifts invited our participants to first remember what data they had available online rather than prompt outright reflection. Then our participants tried to locate where they had displayed said information, as well as pondered their online presentation and how others might perceive them. Besides those thoughts, our participants also reflected on what data they thought they had available online and what someone could find about them online. This awareness may perhaps lead our participants to reconsider how they present themselves online, but also how they preserve their personal data.

Something personal is often understood as meaningful as in the case of the gifts. In this experiment, the aspect of meaningfulness was underlined through the gesture of gifting. While people knew they were part of an experiment, they shared a common understanding of the characteristics of a gift, which was not overshadowed by the fact that those people were strangers to each other. Our participants could not give up their preexisting understanding of the concept of gifting and its meaningful charge.

\subsection{The Experience Behind the Data: Gifts of Biometric Data}

In the second project, [20] (See Figure 3), a tailor-made physicalization of personal data was developed for each of our three participants based on Fitbit ${ }^{1}$ data that was gathered over a period of five weeks. The data gathering month followed individual generative sessions with each participant where we used tangibles in participatory action and discussed our participants' lived experiences behind the data. Data is always situated within a particular type of experience. Having this in mind and since we knew so many of our participants' lived experiences from the data gathering month, we wanted to offer individual gifts that would carry traits from those experiences. More specifically, gifts that would resemble the essence of the project and provoke for one more time the participants' reflections.

\footnotetext{
${ }^{1}$ www.fitibit.com
}

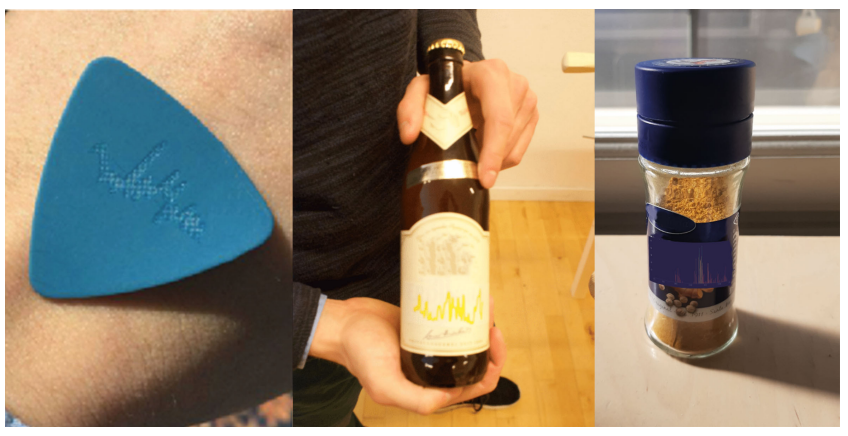

Figure 3: From left to right, Ken's plectrum with his heartbeat data, Kevin's beer bottle with his heartbeat data and Mia's curry with her steps data. The last picture is a conceptual picture of the final design.

Our intention with those objects was to trigger conversations around personal data even after the completion of our research. The gifts resembled agents in the world that would enable storytelling about personal data between the participants and the different people that are in their life and thereby build new experiences. The data agents are everyday objects, which have embedded on their surfaces a data visualization related to a particular moment in the physiological data of our participants. The represented dataset was previously discussed with each of our participants in the generative sessions. The selected data was either representations of some hours of a day or, in one case, of an entire day. Thus, each participant received one data agent tailored to one of their lived experiences. The data agents were given to the participants at the end of the project.

Kevin received a beer bottle (Figure 3, middle) which on its etiquette had a visualization of his heartbeat data. The data was from the next day after he had received two job offers and went out to celebrate with his friends. The celebration included large amounts of alcohol, which resulted in a high heart rate for several hours. The next morning, Kevin received a congratulating virtual reward from Fitbit for being active. Our intention with the data agents was to make him reflect upon that experience and perhaps to provoke thoughts around the impact of alcohol on his body.

While Kevin acknowledged the effects of alcohol, he said that it is a habit that he will not stop. More specifically, he said, "I do think of the effects of alcohol and how unhealthy it might be" [...] "It does make me conscious when I see the data, but it is still something that I do." When we asked him if he would drink the beer later he replied positively. Although we are not aware if our intention to make him reflect went further than the testing room of the physicalization, we do know, through a follow up email, that the beer was not consumed, almost one month after the study was concluded. That indicates that while the data agent was a common product that one can find anywhere (beer), it acquired a special identity that made Kevin to not instantly consume it.

The object that was given to Ken was a plectrum with his heartbeat data on (Figure 3A). The graph was representing three hours of heartbeat data during a concert where Ken was playing bass. His heartbeat rate was unusually high before the concert but also 
while performing. Through the workshop, we found out how important that day was for Ken, therefore our intention was just to observe his reactions and see how he would reflect on the gift. When he received the gift he was very excited and said, "it's like [a] souvenir from the place and time, but not" [...] "It's like a piece of you" (indicating himself) [...] "The closest thing is when you get your tooth pulled out and then you get it" [...] "it's a positive version of that" [...] "you made it for me, but it is me." Regarding Mia the most prominent topic of discussion throughout the study was that she needed to change her lifestyle for one month in order for her stress treatment to be effective. One of the rules she had to follow was to reduce walking which was one of her favourite activities. Thus, as she said during the workshop she provided us with a nonrealistic image of herself over that month of the study, as she said looking at her graph from her time in India "This is not the real me". More specifically, her resting heart rate was lower while she was in India compared to while she was in Denmark. Notably, she said, "the resting heart rate was going down during the weeks I was in India". That was an interesting finding for her as the purpose of her being there was to slow down and "get all the stress out of the body". The illustration of that through the quantified was very surprising, more specifically she said, "you could see it, you could measure it that I was becoming more clam”. As such, a small curry container was given to Mia having on the etiquette a graph of the day with the least amount of steps of the past month as a form of provocation which would reflect her lifestyle in India but while in her home country this time. The design decision to add the data on a curry container came from the fact that food was important during her experience in India "I had a lot of nice food, Indian food, this was very different from what I had at home" but it was also one of her favorite activities similar as walking. Therefore, a curry container which is a product that can be easily associated with India and her number of steps were the two elements that formed the data-agent. Our intention with this gift was to indicate the contradiction between the two life styles in Denmark and India accordingly and prompt her to reflect on that. She was asked to place the gift somewhere at her home and see if it would provoke any reflections or discussions. The reflections we received later from her were related to her experiences using the curry rather than to the data itself. The curry container in this case became a ticket-to-talk about experiences however, not related to the data itself.

Reflecting again on Sherry [34], about how the gifts represent the perception of the donor about the receiver, in our study the data agents were not only about our perception of the identity of the participants. Some of our participants' identity was represented through the data itself that was embedded on the objects. Also, our choices of objects were guided by our participants' lived experiences. So, the data agents, to some extent, were a material representation of our participants' identity representing little of our interpretation of who these people were. The latter, inevitably influenced the identity of the objects. The objects, were not just a beer, a plectrum and curry container anymore, but they were representations of a snapshot in time of our participants' identity, which had material traits.

As Ken describes "it is like a souvenir from the place and time, but not!" Theoretically, these objects constitute the continuation of this

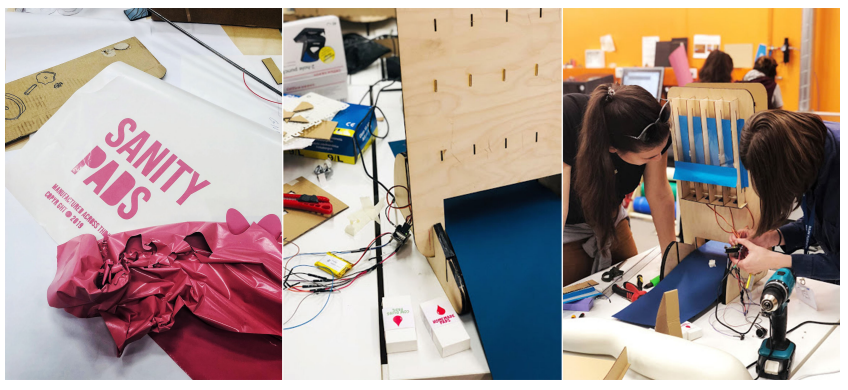

Figure 4: The design process of Sanity Pads. The first picture (from left to right) shows the stamp of the logo on the front layer of the vending machine, second and third picture show assembling the electronics of the prototype.

project. Data agents are objects “from a place and a time," reminding participants some of their experiences. However, they are not only reminders of the experiences that were related to when the data was generated but they were also related to the moment that they received the gift. From that we can speculate that the person looks at the object and remembers the moment that the object was given to them. In a meta-level, the person looks at the graph on the object and they recall the experience of the day the graph was generated. This chain of memories could provoke reflections related to several different experiences, which could be detached from the original experience that the objects were inspired from. The gift-giving was an unrelated experiment to the rest of the project, in the sense that there were not any further intentions rather than triggering reflections. Mostly, it was an expression of gratitude towards the participants.

In that case, the aspect of meaningfulness was triggered first, through the objects itself which was indirectly linked to the lived experience as a reminder, and second, through the data itself, which were actual products of those experiences.

\subsection{Sanity Pads: Sharing Menstrual Experiences}

Zimmerman et al. [44] argue, "Unlike design practice, where the making focuses on making a commercially successful product, design researchers engaged in critical design create artifacts intended to be carefully crafted questions." The third project, Sanity Pads, makes us reflect critically on menstruation hygiene in developing countries, as it juxtaposes a person's menstrual practices with people who are less privileged. This is achieved by creating a speculative product called sanity pads which on each side contains information-including personal information-that act as triggers for thought.

The design of the last project was part of a workshop on Critical Making at UBISS Summer School 2019, which lasted for one week. The workshop explored critical aspects of ubiquitous computing and design. In that context, in a team of three we prototyped a speculative sanitary product called Sanity Pads (see Figure 4). The design process of the Sanity Pads started with an exploration on protests. Our focus was on creating something that would resemble a small protest in everyday life; that would be humoristic; portable; it would call for attention to a lack of access to basic needs; would 


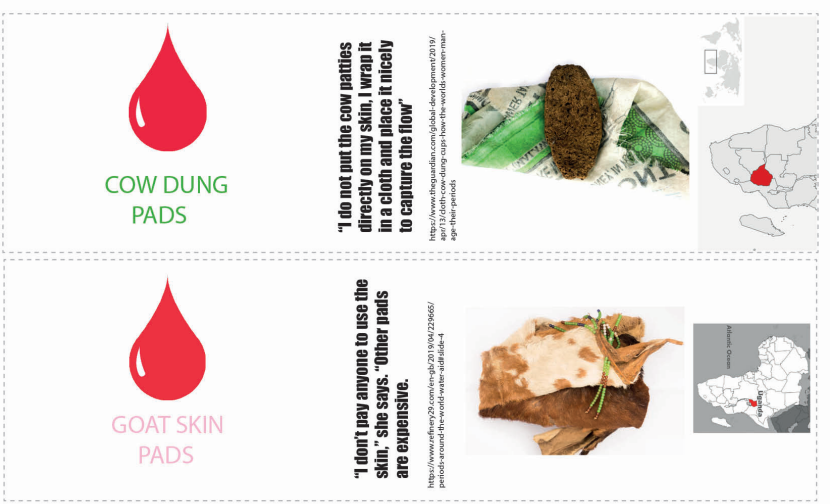

Figure 5: The logo of the Sanity Pads went through two iterations, from a drop icon to the final flower icon. This figure shows two of the initial packages which contained i) personal narratives, ii)map of the countries were the type of Sanitary product was relates to, iii) an image of the menstrual product, and, iv) the logo and name of the product.

raise awareness; and that would empower people to make something small that would have a positive impact in broader terms. Those objectives led to a desk research on relevant social issues the team wanted to explore and a research on the form we wanted our artefact to acquire. Thus, Sanity Pads was created to prompt critical thinking and raise awareness over the types of sanitary products women use around the world, which often may be unhygienic. This is a crucial topic that calls for attention and action as poor menstrual hygiene can cause physical health risks and has been linked to reproductive and urinary tract infections [14]. Besides that, menstruation is an 'issue' that can stop women from reaching their full potential as they miss out on education opportunities crucial to their growth. For example, in Uganda, most adolescent girls who miss school do so because of menstruation-related problems [27].

Linking this back with the rest of our project, here what is meaningful is understood through a wider perspective, as in the case of Mill Road [21]. Sanity Pads reflect an important social issue and through its design call for attention and action hence, this is how it becomes meaningful to people who see it. Nuanced reflection is achieve by synthesizing the information that is displayed on Sanity pads with our own individual experiences with menstruation and hygiene. Baumer et al. [4] argue that to achieve nuanced reflection, data must be synthesized, not simply encountered. This is something that Sanity Pads facilitate. For some it may be shocking, while for others it may be more familiar. During our design process we found different ways through which women deal with their menstruation in different sides of the planet. Some of those were cow dung, to pure cotton, and soil placed in fabric. Therefore, we decided to display some of those actual 'products' as data agents.

We prototyped a vending machine (Figure 6), which would allow people to receive our speculative product. Vending machines with hygiene products are typically found in public bathrooms for people to access the products when they need them. To gain more inspiration on how our vending machine would look like we searched online for different examples borrowing different characteristics

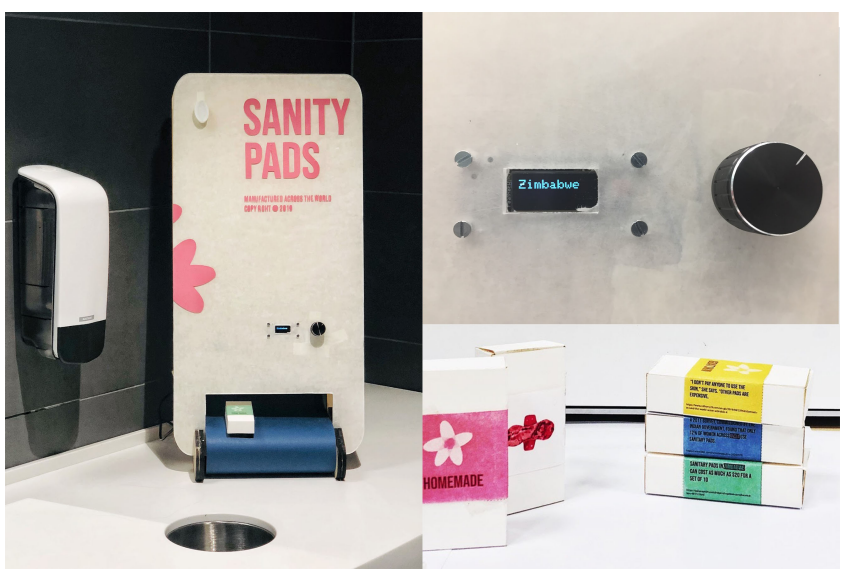

Figure 6: The final prototype of the vending machine, a close up of the display it had on top and the boxes that contained the sanity pads.

from them. Our vending machine consisted of a small display, a knob through which people could navigate through different options and a button to dispense the product. To operate the vending machine people had to navigate through different countries. After the person had decided on the country, they could confirm their choice by pressing a button that was on the top left corner of the vending machine. Then the Sanity Pads product would be dispensed through the lower part of the vending machine. Depending on the country of choice, the product would show on its surface four elements(Figure 5), one on each side of the box. Those elements were: a) the logo of our product under which was written the type of sanitary product women use in the region of choice, b) the map of the country of origin of each particular sanitary product, c) a picture of the product itself and last, d) personal information or other data that was related to menstrual hygiene of different women in each region. For instance, in Figure 5, the information that appears on the side of one of the boxes comes from a woman who has experienced using unhygienic products during menstruation. Notably, she said "I don't pay anyone to use the skin"-as in her country she used animal skin as pads during menstruation-she says "other pads are expensive." This brings the personal narrative into the objects, similarly to the other two case studies we have presented in this paper where personal data/information is linked with everyday objects.

Baumer [3] suggests that instead of designing systems to make people more reflective we should instead focus on how we can deepen our understandings of reflection through those projects. "Thus, avoiding an insistence on demonstrating that our design 'works' may open up opportunities to understand more fully both the nature of reflection and the potential impacts of our intervention." [3] Our prototype was a critical design intervention whose purpose was to shake the normative by making people reflect on their own reality through the lens of others. The example of Sanity Pads could have been a product in the world to raise awareness about other people around us yet reflected through our own personal daily routine. After all, the purpose of this project is to create moments of 
perplexity [3] and make us think about the wider social and ethical aspects of women's hygiene around the world through which it becomes meaningful to people.

\section{UNDERSTANDING THE DATA AGENTS}

In this section, we use Fleck and Fitzpatrick's reflection framework [12] and Mekler and Hornbæk's components of the experience of meaning [26] to reflect on how data agents prompted self-reflection via affording meaningfulness. While in [12] each level of reflection builds upon the next, in [26], the components of meaning are interconnected, with connectedness being a starting point and a prerequisite for the other four.

\subsection{Objects and Data Connect to Memories}

Across our three projects the data agents acted as triggers to remember a particular situation. Specifically, following Mekler and Hornbæk's notion of connectedness [26], data agents connected to our participants' personal memories and experiences. While these acts of reminiscing are not forcibly reflective (R0) [12], data agents made participants' previous experiences available to further reflection. For instance, Kevin's beer bottle was associated with his memories of the job offers and resulting celebration. While he expressed no intent to stop drinking, the heart rate data still served as a reminder of past excesses. As such, the role of the data representations (or indirectly of the personal data in case of the first project) is much more subtle compared to the higher levels of reflection, where the data begins to take an active role in the reflective process.

\subsection{Data Shapes the Agency of the Object}

Beyond reminiscence, data agents also got our participants to "think about what they are doing and provide justifications or explanations for knowledge, actions or events." (R1) [12, p. 219] Objects and data did not only individually connect to participants' memories and experiences, but also implied connectedness among each other, thus 'asking' participants to make sense of how object and data fit into their life (i.e., coherence [26]). For example, while the Jazz CD Alice received was linked to her obvious interest in music, it got her to reflect on what personal data she had made publicly available, as well as how Jack managed to find out about her interests and decide on a gift. As such, the type of data and the objects act together to prompt a synthesis of different events that lead to reflection.

Notably, while all our studies originated from the idea of selfagain channeling connectedness [26]-the presence of other people also shaped how our participants understood themselves. Indeed, Fleck and Fitzpatrick argue that "the presence of another person is also beneficial in encouraging the giving of justifications or explanations, as it makes sense to explain things to other people, especially if they do not share the same knowledge, understanding or experience as you." [12, p. 219] For instance, in the case of the two first projects, the self is projected through the lens of a stranger, which makes the person reflect on how others may perceive them. Similarly, the data agents were also gifted by us to our participants. Our data gifts reflected the lived experiences behind our participants' personal data, but at the same time they were also linked to us. In that sense, we supported our participants' self-reflections indirectly. The sanity pads did so differently, in that they use others' personal narratives to make people reflect on their practices and extend them to their self. In that way, the data agents take up multiple roles during the reflective process. Those roles may be raising reflecting questions, but also imply indirectly the presence of a third person to initiate the reflective process.

\subsection{Reviewing the Data in Different Environments}

According to Fleck and Fitzpatrick [12], providing people with a different perspective on information, as well as affording opportunities for reviewing said information more than once will encourage dialogic reflection (R2). The reviewing of historical data [11] on an everyday object that actually implied use, suggests that that data would be reviewed by the person under different circumstances and in different environments. We connect this process again to coherence as the reading of data in different environments can provide people with moments in which data and environment would suddenly make sense and would allow seeing the data through different perspectives. The data agents were artifacts small in size, which made them easy to transport to different environments, as well as tied to aspects that were personally meaningful e.g., Mia's curry container was tied to her goals to move more, and her experience with food in India. This might have facilitated our participants to view their data through different perspectives. For instance, the data-gifts supported storytelling in mundane environments while being exhibited in someone's apartment. In the case of Sanity Pads, for instance, the type of data they illustrate contains aspects of narration. This element of easy mobility can also be seen as a supportive element during the reflective process as Fleck and Fitzpatrick argue, "Simulation environments [...] can also support reflection through allowing the quick and relatively cost free exploration of these environments, allowing participants to experience different possible outcomes." In our work due to the size of data agents they could be actually transported to different physical environments where they could trigger reflection.

\subsection{Personal Data and the World}

Higher levels of reflection (R3 and R4) are rare, because they suggest a fundamental transformation in the way of thinking [12]. Reaching such higher levels of reflection also imply a broader understanding of the person and the world, which we believe to have touched upon through our last project, the Sanity Pads: Transformative and Critical Reflection. We can speculate that the Sanity Pads is a 'product', which can suggest action by creating a need to help other people around the world but always by enabling a comparison, that goes beyond the immediate context, between a privileged self and others who do not have the luxury to use hygienic products. In this case the aspects of significance, connectedness, and purpose play an important role. From that, we speculate that meaningful experiences that create some kind of comparison between the person and the world are more likely to support higher levels of reflection. This can also be understood when compared with previous work on data physicalization [e.g., 16, 29], where people are more likely to reach up until the second level of reflection. A resonance between the object and the data is necessary for it to be meaningful for the person. 
Table 3: Illustration of relationships between the two concepts.

\begin{tabular}{ccl}
\hline Reflection Levels & Components of Meaning & Characteristics of data agents \\
\hline R0 & connectedness & form of object set the stage \\
R1 & connectedness, coherence & interplay between form and data \\
R2 & coherence & portability, role of others \\
R3 and R4 & connectedness, significance, purpose & form and data resonance \\
\hline
\end{tabular}

Above, we have created four themes through which we illustrate how we have understood potential relationships between meaningfulness and reflection. While those themes are tightly related to our projects, we see them as relevant also for other works around meaningful personal data representations. In the first theme, we argue that the type of object on which personal data is illustrated can act as triggers for reminiscence and constitute the basis for reflection, in that case we link connectedness to the first level of reflection (R0). Appropriate combinations of each specific format (type of everyday object that the person can relate to) with personal data, may enable reflective thinking and as such, it was associated with the second level of reflection (R1) and was linked to connectedness and coherence. We connected the third level of reflection (R2) again with coherence as the flexibility of data agents in size could make them platform for reflection in different contexts through which people may achieve sense-making. Finally, we relate the fourth (R3) and fifth (R4) levels of reflection to connectedness, significance, and purpose as meaningful experiences that are linked to personal data can facilitate comparison between the person and the world and are more likely to enable the higher levels of reflection. Table 3 shows the relationships we found as illustrated above.

Based on the framework of meaning [26], resonance cannot be associated to any level of reflection as it denotes 'the immediate, unreflected experience of something making sense, without the need to reflect on why or how it does so.' However, resonance is a component of meaning that we found in our second project when Ken received his data-agent, the plectrum. The moment Ken received the plectrum, he expressed out loud his thoughts trying to illustrate the relationship he felt he had with that object. As he notably said, "The closest thing is when you get your tooth pulled out and then you get it" [...] "it's a positive version of that" [...] "you made it for me, but it is me" indicating a special fit or connection. In that sense, Ken's experience could be link to resonance, as the data-agent just felt right, it was perceived as a part of himself and could be associated with the 'feeling of meaning', something that could not be explained but resonated with the self.

\section{CONCLUSION}

While the mapping above nicely illustrates the depth of meaningfulness that connects to each of the data agents, Carpenter's [7] work can provide us with some insights into how the data agents were meaningful to people focusing on the different characteristics of the objects. All of our three projects touched upon different aspects of meaningfulness [7] through representations of personal data on everyday objects which are tangible, non-screen and some of which incorporate characteristics of crafts. In our work, we aimed for our data agents to become part of people's everyday life in triggering self-reflection. The physicality/tangibility of the artifacts made them more visible and significant than ordinary digital data representations, as all of them are everyday objects (e.g., the guitar plectrum), so they coherently fit into our participants' lives (Ken's identity) and might serve a personally important purpose (playing bass). In our second project, data gifts and physiological data, the data visualizations have been added to the object, making the object unique and asking for participants' attention while borrowing characteristics of crafts. Last, while screens may take our attention away from ourselves and our environments, data agents instead strengthen this connectedness to our surroundings allowing the person to focus on the lived experience.

While our projects are not related to technological artifacts, they suggest a path towards exploring data representations as part of everyday life. They make us think how we can create more reflective and meaningful technologies where data does not have the characteristics of simply giving quantitative insights but it is considered from an experience-centric point of view [43] where it is perceived e.g. as a 'totem' [31] of a particular experience and it can become a ticket-to-talk among different people. We believe it is rather essential to begin to discover new aspects of how technology can contribute to a more holistic understanding of the notion of personal data, which includes seeing information as meaningful and significant-as if personal data were mementos people keep over time. Of course, we do not suggest that people should stop in everyday life and engage with data, we rather imply that one's personal information should begin to become part of their environments in meaningful ways for the person. As Mekler and Hornbæk argue, "Meaning is a complex phenomenon." [26] In our work we showed how meaning and self-reflection are interrelated but we also argued that meaningfulness is an important component, which can lead to the highest levels of reflection underlining that we should begin to consider infrastructures for meaningful data. For instance, as argued by Taylor and Harper [37], there are already aspects of gifting (meaningfulness) in how people treat messages that are significant for them. We argue that those types of personal data are the ones that require more of our attention in designing for meaningfulness.

In this paper we have presented three different projects where personal data have been coupled with everyday objects. To understand how people made sense of those objects and how that might have led to self-reflections, we used two different frameworks to help us create links between reflection and meaningfulness. We do not suggest that our findings are readily generalizable, but they illustrate a first step towards understanding the relationship between meaning-making and self-reflection in the context of personal data representations and open up the topic for further discussion in the HCI community. 


\section{ACKNOWLEDGMENTS}

We would like to acknowledge the support of Eric Paulos, Jill Miller and Georgi V. Georgiev who were the instructors of the CRITICAL MAKING: DESIGNING FOR ACTIVISM workshop. Last, thank you to Kailin Li and Patrycja Zdziarska who were part of the design and realization of Sanity Pads.

\section{REFERENCES}

[1] Jeffrey Bardzell and Shaowen Bardzell. 2013. What is" critical" about critical design?. In Proceedings of the SIGCHI conference on human factors in computing systems. ACM, Paris, 3297-3306.

[2] Stephen Barrass. 2014. Acoustic sonification of blood pressure in the form of a singing bowl. In Conference on Sonification of Health and Environmental Data (SoniHED). SoniHED, University of York, 1-6.

[3] Eric PS Baumer. 2015. Reflective informatics: conceptual dimensions for designing technologies of reflection. In Proceedings of the 33rd Annual ACM Conference on Human Factors in Computing Systems. ACM, Korea, 585-594.

[4] Eric PS Baumer, Vera Khovanskaya, Mark Matthews, Lindsay Reynolds, Victoria Schwanda Sosik, and Geri Gay. 2014. Reviewing reflection: on the use of reflection in interactive system design. In Proceedings of the 2014 conference on Designing interactive systems. ACM, Vancouver, 93-102.

[5] Laurens Boer and Jared Donovan. 2012. Provotypes for participatory innovation. In Proceedings of the designing interactive systems conference. ACM, Newcastle, 388-397.

[6] Jerome Seymour Bruner. 1990. Acts of meaning. Vol. 3. Harvard university press, US.

[7] Vanessa Carpenter. 2019. Designing for Meaningfulness in Future Smart Prod ucts: an explorative investigation of values and physical characteristics. Ph.D. Dissertation. Aalborg Universitetsforlag.

[8] Eun Kyoung Choe, Nicole B Lee, Bongshin Lee, Wanda Pratt, and Julie A Kientz. 2014. Understanding quantified-selfers' practices in collecting and exploring personal data. In Proceedings of the SIGCHI Conference on Human Factors in Computing Systems. ACM, Toronto, 1143-1152.

[9] Anthony Dunne. 2008. Hertzian tales: Electronic products, aesthetic experience, and critical design. The MIT press, London.

[10] Anthony Dunne and Fiona Raby. 2001. Design noir: The secret life of electronic objects. Springer Science \& Business Media, London.

[11] Chris Elsden, David S Kirk, and Abigail C Durrant. 2016. A quantified past: Toward design for remembering with personal informatics. Human-Computer Interaction 31, 6 (2016), 518-557.

[12] Rowanne Fleck and Geraldine Fitzpatrick. 2010. Reflecting on reflection: framing a design landscape. In Proceedings of the 22nd Conference of the Computer-Human Interaction Special Interest Group of Australia on Computer-Human Interaction. ACM, Brisbane, 216-223.

[13] Laurie Frick. 2011. Sleep Patterns. Frick. Retrieved September 9, 2019 from https://vimeo.com/21852158

[14] Julie Hennegan, Catherine Dolan, Maryalice Wu, Linda Scott, and Paul Montgomery. 2016. Measuring the prevalence and impact of poor menstrual hygiene management: a quantitative survey of schoolgirls in rural Uganda. BMF open 6 , 12 (2016), e012596.

[15] Steven Houben, Connie Golsteijn, Sarah Gallacher, Rose Johnson, Saskia Bakker Nicolai Marquardt, Licia Capra, and Yvonne Rogers. 2016. Physikit: Data engagement through physical ambient visualizations in the home. In Proceedings of the 2016 CHI Conference on Human Factors in Computing Systems. ACM, San Jose, 1608-1619.

[16] Samuel Huron, Sheelagh Carpendale, Alice Thudt, Anthony Tang, and Michael Mauerer. 2014. Constructive visualization. In Proceedings of the 2014 conference on Designing interactive systems. ACM, Vancouver, 433-442.

[17] Yvonne Jansen, Pierre Dragicevic, Petra Isenberg, Jason Alexander, Abhijit Karnik, Johan Kildal, Sriram Subramanian, and Kasper Hornbæk. 2015. Opportunities and challenges for data physicalization. In Proceedings of the 33rd Annual ACM Conference on Human Factors in Computing Systems. ACM, Seoul, 3227-3236.

[18] Loizeau Jimmy and Auger James. 2002. Audio tooth implant. Goldsmiths University of London. http://research.gold.ac.uk/id/eprint/289/

[19] Maria Karyda, Iyubanit Rodríguez, and Andrés Lucero. 2018. Gifting interpretations of personal data. In Extended Abstracts of the 2018 CHI Conference on Human Factors in Computing Systems. ACM, Montreal, 1-6.

[20] Maria Karyda, Danielle Wilde, and Mette Kjærsgaard. 2020. Narrative Physicalization: Supporting Interactive Engagement with Personal Data. IEEE Computer Graphics and Applications? (2020), 1-14.

[21] Lisa Koeman, Vaiva Kalnikaité, and Yvonne Rogers. 2015. " Everyone Is Talking about It!" A Distributed Approach to Urban Voting Technology and Visualisations.
In Proceedings of the 33rd Annual ACM Conference on Human Factors in Computing Systems. ACM, Seoul, 3127-3136.

[22] Ilpo Koskinen, John Zimmerman, Thomas Binder, Johan Redstrom, and Stephan Wensveen. 2011. Design research through practice: From the lab, field, and showroom. Elsevier, Netherlands.

[23] Ian Li, Anind Dey, and Jodi Forlizzi. 2010. A stage-based model of personal informatics systems. In Proceedings of the SIGCHI conference on human factors in computing systems. ACM, Atlanta, 557-566.

[24] Ian Li, Anind K Dey, and Jodi Forlizzi. 2011. Understanding my data, myself: supporting self-reflection with ubicomp technologies. In Proceedings of the 13th international conference on Ubiquitous computing. ACM, Beijing, 405-414.

[25] Deborah Lupton. 2017. Feeling your data: Touch and making sense of personal digital data. New Media \& Society 19, 10 (2017), 1599-1614.

[26] Elisa D. Mekler and Kasper Hornbæk. 2019. A Framework for the Experience of Meaning in Human-Computer Interaction. In Proceedings of the 2019 CHI Conference on Human Factors in Computing Systems (Glasgow, Scotland Uk) (CHI '19). Association for Computing Machinery, New York, NY, USA, Article 225, 15 pages. https://doi.org/10.1145/3290605.3300455

[27] George Miiro, Rwamahe Rutakumwa, Jessica Nakiyingi-Miiro, Kevin Nakuya, Saidat Musoke, Juliet Namakula, Suzanna Francis, Belen Torondel, Lorna J Gibson, David A Ross, et al. 2018. Menstrual health and school absenteeism among adolescent girls in Uganda (MENISCUS): a feasibility study. BMC women's health 18, 1 (2018), 4.

[28] Andrew Vande Moere. 2008. Beyond the tyranny of the pixel: Exploring the physicality of information visualization. In 2008 12th International Conference Information Visualisation. IEEE, London, 469-474.

[29] Bettina Nissen and John Bowers. 2015. Data-things: digital fabrication situated within participatory data translation activities. In Proceedings of the 33rd Annual ACM Conference on Human Factors in Computing Systems. ACM, Seoul, 24672476.

[30] Andrew Vande Moere Pierre Dragicevic, Yvonne Jansen. 2019. Data Physicalization. Springer Handbook of Human Computer Interaction, Netherlands, 1-50.

[31] John Rooksby, Mattias Rost, Alistair Morrison, and Matthew Chalmers. 2014. Personal tracking as lived informatics. In Proceedings of the SIGCHI conference on human factors in computing systems. ACM, Toronto, 1163-1172.

[32] Donald A Schön. 1987. Educating the reflective practitioner. Jossey-Bass, San Francisco.

[33] Mark Selby and David Kirk. 2015. Experiential manufacturing: The earthquake shelf. In Proceedings of the 2nd Biennial Research Through Design Conference. RTD, Cambridge,UK, 25-27.

[34] John F Sherry Jr. 1983. Gift giving in anthropological perspective. fournal of consumer research 10, 2 (1983), 157-168.

[35] Ricardo Sosa, Victoria Gerrard, Antonio Esparza, Rebeca Torres, Robbie Napper, et al. 2018. Data Objects: Design Principles For Data Physicalisation. In DS 92: Proceedings of the DESIGN 2018 15th International Design Conference. DS, Croatia, 1685-1696.

[36] Simon Stusak, Aurélien Tabard, Franziska Sauka, Rohit Ashok Khot, and Andreas Butz. 2014. Activity sculptures: Exploring the impact of physical visualizations on running activity. IEEE Transactions on Visualization and Computer Graphics 20,12 (2014), 2201-2210.

[37] Alex S Taylor and Richard Harper. 2002. Age-old practices in the'new world' a study of gift-giving between teenage mobile phone users. In Proceedings of the SIGCHI conference on Human factors in computing systems. ACM, Minneapolis, 439-446.

[38] Lisa Thomas, Pam Briggs, Finola Kerrigan, and Andrew Hart. 2018. Exploring digital remediation in support of personal reflection. International fournal of Human-Computer Studies 110 (2018), 53-62.

[39] Alice Thudt, Uta Hinrichs, and Sheelagh Carpendale. 2017. Data craft: integrating data into daily practices and shared reflections. 2017 CHI Conference on Human Factors in Computing Systems. CHI2017Workshop,http://alicethudt.de/datacraft/

[40] Alice Thudt, Uta Hinrichs, Samuel Huron, and Sheelagh Carpendale. 2018. Selfreflection and personal physicalization construction. In Proceedings of the 2018 CHI Conference on Human Factors in Computing Systems. ACM, Montreal, 1-13.

[41] Kendra Wannamaker, Wesley J Willett, Lora A Oehlberg, and Sheelagh Carpendale. 2019. Data Embroidery: Exploring Alternative Mediums for Personal Physicalization. PRISM, Calgary, ? https://prism.ucalgary.ca/handle/1880/110218

[42] Gary Wolf. 2009. Know thyself: Tracking every facet of life, from sleep to mood to pain. Wired. https://www.wired.com/2009/06/lbnp-knowthyself/

[43] Peter Wright, John McCarthy, and Lisa Meekison. 2003. Making sense of experience. Springer, Dordrecht, 43-53.

[44] John Zimmerman, Jodi Forlizzi, and Shelley Evenson. 2007. Research through design as a method for interaction design research in HCI. In Proceedings of the SIGCHI conference on Human factors in computing systems. ACM, San Jose, 493-502. 